\title{
A Universal Equation Governing Engineering Stress-strain Curves of Polycrystals
}

\author{
Nam Hoe HEO, ${ }^{*}$ Yoon-Uk HEO and Sung-Joon KIM \\ Graduate Institute of Ferrous Technology, Pohang University of Science and Technology, Pohang, $790-784$ Republic of Korea. \\ (Received on December 18, 2015; accepted on February 9, 2016)
}

\begin{abstract}
A universal equation for understanding various phenomena observed on the engineering stress-strain curves of polycrystals, which includes the Hall-Petch relation concepts, is derived. The derivation of the equation begins from the simple condition that the given constant strain rate is the sum of the elastic strain rate and the plastic strain rate. The physical meanings for the various phenomena on engineering stress-strain curves are analyzed from the viewpoint of the competition between two strain rates.
\end{abstract}

KEY WORDS: universal equation; yield drop; Lüders band; work hardening; Hall-Petch relation.

\section{Introduction}

Among several phenomena on a schematic engineering stress-strain curve of Fig. 1, the yield point (point $a$ ) and the upper yield point (point $b$ ) have been simply defined by

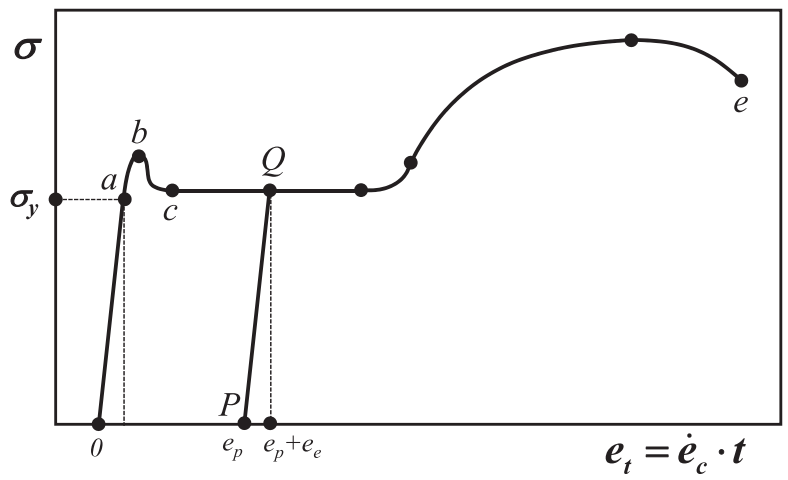

Fig. 1. A schematic engineering stress-strain curve of a polycrystalline metal. Symbols $e_{t}, e_{p}, e_{e}$, and $\dot{e}_{c}$ are the total strain which is the sum of the plastic strain $e_{p}$ and the elastic strain $e_{e}$ and the constant strain rate, respectively. a deviation point from the elastic region and a point where the slope of the stress-strain curve goes to zero, respectively. The yield drop phenomenon (the region of $b c$ ) in Fig. 1 is frequently observed in annealed BCC metals. As shown in Fig. 2, the magnitude of yield drop increases with decreasing grain size ${ }^{1,2)}$ and with increasing strain rate. ${ }^{3)}$ The Lüders strain in the Fe-21Ni steel of Fig. 2(a) which is composed of ferrite and austenite increases with decreasing grain size to a critical grain size $(0.57 \mu \mathrm{m})$, after which the Lüders strain decreases rather with decreasing grain size. In an interstitialfree steel of Fig. 2(b), such a tendency of Fig. 2(a) is not strong. It is however expected in Fig. 2(b) that a maximized Lüder strain may be observed in a critical grain size between $0.8-1.6 \mu \mathrm{m}$. As shown in Fig. 2(b), the overall work hardening behavior decreases overall with decreasing grain size.

It has been proposed by Cottrell ${ }^{4}$ ) that the yield drop phenomenon is a result of breaking away of mobile dislocations from the Cottrell atmosphere formed around the dislocations, although it is still in doubt. The Johnston-Gilman theory ${ }^{5,6)}$ has suggested how the rapid dislocation multiplication at the beginning of a tensile test can produce the yield
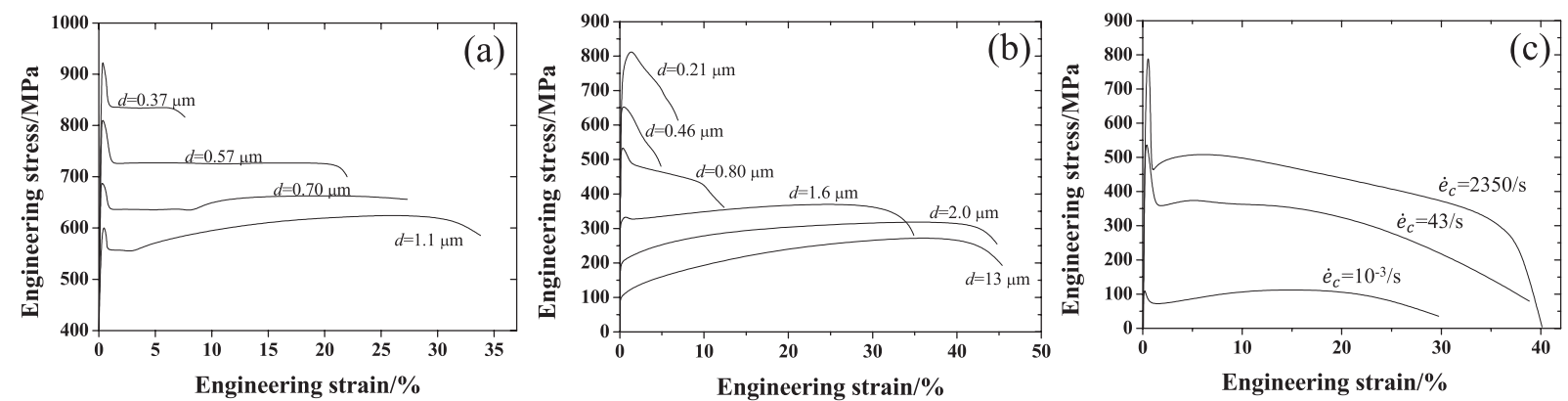

Fig. 2. The dependence of stress-strain curve on grain size and strain rate: (a) Fe-21Ni steel, ${ }^{1)}$ (b) interstitial-free steel, ${ }^{2)}$ and (c) pure iron. ${ }^{3)}$ The original curves of Fig. 2(b) have been redrawn as the engineering stress-strain curves.

\footnotetext{
* Corresponding author: E-mail: nhheo@postech.ac.kr

DOI: http://dx.doi.org/10.2355/isijinternational.ISIJINT-2015-719
} 
drop, while they do not account for the Lüders strain which was noted more than a century ago. ${ }^{7)}$ The refined grain size is usually preferred for the high strength and toughness of structural materials. The yield strength increases with decreasing grain size. As shown in Figs. 2(a) and 2(b), the ductility decreases however abruptly with decreasing grain size. Such a low ductility of nanocrystalline materials has been mainly attributed to such defects as pores remaining within the materials during processing. ${ }^{8-16)}$

In spite of the long time research, several unsolved intrinsic questions for engineering stress-strain curves have not been clarified so far: yielding phenomenon, the existence or the absence of yield drop that depends on materials, the reason why the yield drop magnitude is changed with temperature and strain rate, the dependence of Lüders strain on grain size, strain ageing, and the low ductility in nanocrystalline materials.

In order to understand fundamental concepts implicit in engineering stress-strain curves of polycrystals, a universal equation governing the engineering stress-strain curves is first derived. The physical meanings of the various phenomena are understood on the basis of the equation including the Hall-Petch relation concept.

\section{Derivation of Equation Governing Engineering Stress-strain Curves of Single Crystals}

At a point $Q$ on the engineering stress-strain curve of Fig. 1 , the total strain $e_{t}$ is the sum of the plastic strain $e_{p}$ and the elastic strain $e_{e}$. When the tensile test is performed at the constant strain rate $\dot{e}_{c}$, the total strain rate $\dot{e}_{t}$ at a point on the engineering stress-strain curve, which is equal to the constant strain rate $\dot{e}_{c}$, is always the sum of the plastic strain rate $\dot{e}_{p}$ and the elastic strain rate $\dot{e}_{e}$. In a true stress-strain curve, the total true strain rate $\dot{\varepsilon}_{t}$ is not equal to $\dot{e}_{t}$ or $\dot{e}_{c}$. This is because the total true strain $\varepsilon_{t}$ is expressed by $\varepsilon_{t}=$ ln $\left(e_{t}+1\right)$ and so $\dot{\varepsilon}_{t}=\frac{1}{e_{t}+1} \cdot \dot{e}_{t}$. There, the total true strain rate $\dot{\varepsilon}_{t}$ is not constant but decreases gradually with increasing total engineering strain.

Because the diminution in elastic modulus of polycrystalline metals by plastic deformation is at best $6-11 \%$ of the initial value, ${ }^{17,18)}$ it is hereafter assumed that the elastic modulus $E$ is not changed but constant during plastic deformation in order to simplify the explanation below. The line $O a$ of Fig. 1 is therefore parallel to the line $P Q$. It is emphasized that the flow stress at the point $Q$ is the yield strength of the specimen pre-strained to the point $Q$. That is, if the load is removed, the flow stress at the point $Q$ decreases to zero, following the line $Q P$. If the load is again applied to the pre-strained specimen without strain aging, the stress increases to the point $Q$, following the same $P Q$ line. After the point $Q$, the stress-strain curve follows the curve $Q e$ with increasing total strain. Such an elastic stress-strain relation at an arbitrary point on the stress-strain curve is expressed by

$$
\sigma\left(e_{t}\right)=E \cdot e_{e}
$$

From the relations of $\sigma=\sigma\left(e_{t}\right)$ and $e_{t}=\dot{e}_{c} \cdot t$, the derivative of $\sigma\left(e_{t}\right)$ by $e_{t}$ is expressed by

$$
\frac{d \sigma\left(e_{t}\right)}{d e_{t}}=\frac{1}{\dot{e}_{c}} \cdot \frac{d \sigma\left(e_{t}\right)}{d t}
$$

From Eqs. (1) and (2), the elastic strain rate at time $t$ is finally expressed by

$$
\dot{e}_{e}=\frac{\dot{e}_{c}}{E} \cdot \frac{d \sigma\left(e_{t}\right)}{d e_{t}}
$$

Within the elastic region $0 a$ of Fig. 1, the derivative $\frac{d \sigma\left(e_{t}\right)}{d e_{t}}$ is the elastic modulus $E$ due to no plasticity. Within the region, the elastic strain rate $\dot{e}_{e}$ equals the given constant strain rate $\dot{e}_{c}$ and it is highest on the engineering stress-strain curve.

Meanwhile, the plastic strain rate ${ }^{5,6)}$ of a single crystal is given by

$$
\dot{e}_{p}=\frac{1}{2} \boldsymbol{b} \rho_{m} v
$$

where $\rho_{m}$ is the mobile dislocation density within the single crystal, $\boldsymbol{b}$ is the Burgers vector, and $v$ is the dislocation velocity which is equal to $\left(\tau / \tau_{o}\right)^{n}$. Here, $\tau$ is the resolved shear stress produced on the slip plane of the single crystal by the applied stress, $\tau_{o}$ is the shear stress that gives a unit velocity (usually, $1 \mathrm{~cm} / \mathrm{sec}$ ) of the mobile dislocations, and $n$ is the velocity stress exponent. The constant strain rate, which is the sum of the elastic and the plastic strain rates, is, as a result, expressed by

$$
\dot{e}_{c}=\frac{\dot{e}_{c}}{E} \cdot \frac{d \sigma\left(e_{t}\right)}{d e_{t}}+\frac{1}{2} \rho_{m} \boldsymbol{b}\left(\frac{\tau}{\tau_{o}}\right)^{n}
$$

As the derivative $\frac{d \sigma\left(e_{t}\right)}{d e_{t}}$ term of Eq. (5) decreases, the elastic strain rate $\dot{e}_{e}$ decreases, while the plastic strain rate increases to maintain the constant strain rate $\dot{e}_{c}$. Due to the increase in immobile dislocation density during plastic deformation, $\tau_{o}$ is not constant in a material, ${ }^{19)}$ and it increases with increasing plastic deformation. A similar form to Eq. (5) has been also derived, in order to explain the yield drop phenomenon in a single crystal. ${ }^{20)}$ However, this model is based on two decisive errors where $\tau_{o}$ is constant and the incremental portion of flow stress is linearly proportional to the plastic strain. Unlike the research ${ }^{3)}$ which shows the abrupt increase in yield drop magnitude with increasing strain rate, the model causes, as a result, little increase in yield drop magnitude with increasing strain rate. Furthermore, the model is not applicable to single crystals showing the Lüders strain where the flow stress is not changed with plastic strain.

\section{Derivation of Hall-Petch Relation for Yield Strength and Understanding of Coefficient and Friction Stress}

Before the application of the Hall-Petch relation to the derivation of a universal equation governing engineering stress-strain curves of polycrystals, the Hall-Petch relation for the yield strength is first derived. Using Fig. 3(a), the Hall-Petch relation for the yield strength of polycrystals is 
directly derived from the Smith's equation ${ }^{21)}$ which is based on the dislocation pile-up model and given by

$$
\begin{aligned}
\sigma_{s}= & (1 / 2) \cdot\left(L_{o} / r\right)^{1 / 2} \cdot\left(\sigma_{a}-\sigma_{y o}\right) \cos \alpha \cos \beta \\
& \cdot\{2 \cos (3 \theta / 2)+\sin \theta \sin (\theta / 2)\}
\end{aligned}
$$

In Fig. 3(a), $\sigma_{\mathrm{s}}$ is a shear stress normal to the point $M$ at a distance $r$ from the front of pile-up edge dislocations by the applied stress $\sigma_{a} . L_{o}(=d / 2)$ where $d$ is the effective grain size is the distance from the Frank-Reed source located at the center of the grain to the front of pile-up edge dislocations. $\sigma_{y o}$ in Eq. (6) is the minimum applied stress necessary for the movement of mobile dislocations, $\cos \alpha \cos \beta$ is the Schmid factor, $\theta$ is an angle between the segment $r$ and the slip direction. The stress $\sigma_{s}$ decreases abruptly with increasing $r$. Because the region around the point $M$ is mobile dislocation-free due to the extremely high stress field generated at the front of pile-up dislocations, the region can be treated as a nearly perfect crystal. In a randomly oriented polycrystalline metal, grains show various orientations to the applied stress $\sigma_{a}$. Among these grains, specific grains can show an orientation favorable for slip against the applied stress where $\alpha=\beta \cong 45^{\circ}$ and $\cos \alpha \cos \beta \cong 0.5$. The term $2 \cos (3 \theta / 2)+\sin \theta \sin (\theta / 2)$ in Eq. (6) increases with decreasing $\theta$. Considering the pile-up of dislocations, Eq. (6) is defined at an angle larger than $10^{\circ}$ which is the minimum angle forming a high angle grain boundary between the grains. ${ }^{22)}$

During tensile test, the mobile dislocations move first on the slip plane of the specific grains, when the resolved shear stress $\tau$ formed on the slip plane of the specific grains exceeds the critical resolved shear stress $\tau_{\text {crss }}$. In this case, the stress $\sigma_{y o}$ of Fig. 3 is equal to two times of the critical resolved shear stress $\tau_{\text {crss }}$ which is the minimum shear stress necessary for the movement of mobile dislocations.

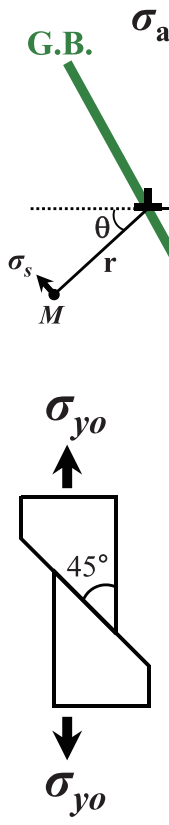

(b) (a)

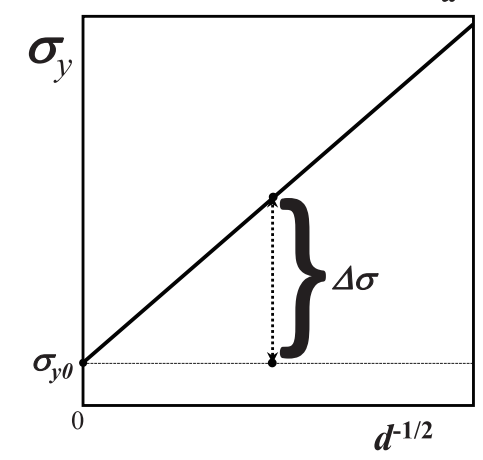

(c)
Fig. 3. Schematic diagrams for deriving the Hall-Petch relation for the yield strength (a), minimum yield strength of single crystal (b), and physical meanings of $\sigma_{y o}$ and $\Delta \sigma$ (c). (Online version in color.)
As the applied stress increases, the mobile dislocations pile up at the grain boundaries of the specific grains. Yielding occurs at the instance that the pile-up dislocations propagate onto the slip plane of the neighboring grains, resulting in the deviation from the elastic region of the engineering stress-strain curve. More analytically speaking, yielding occurs, when the stress $\sigma_{s}$ formed at the front of the mobile edge dislocations piled up at the specific grain boundaries exceeds roughly the theoretical shear strength $\sigma_{\text {thsh }}$ between the slip planes of the neighboring grains treated as perfect crystals, which is expressed by

$$
\sigma_{t h s h}=\frac{G \boldsymbol{b}}{2 \pi l}=\frac{E \boldsymbol{b}}{4 \pi(1+v) \cdot l}
$$

where $G$ is the shear modulus in the slip direction, $\boldsymbol{b}$ is the Burgers vector, $l$ is the interplanar spacing between the slip plane of the neighboring grains, $E$ is the elastic modulus in the slip direction, and $v$ is the Poisson's ratio. The HallPetch relation for yield strength can be therefore obtained from the combination of Eqs. (6) and (7). As a result, the Hall-Petch coefficient $k$ in Fig. 3(c) is proportional to the elastic modulus in the slip direction. The stress $\sigma_{y o}$ of Fig. 3(c), which is the yield strength of the polycrystal of infinite grain size, is also equal to the yield strength of the single crystal under the condition of Schmid factor of 0.5 , as shown in Fig. 3(b). The applied stress component $\Delta \sigma$ in a grain size $d$ of Fig. 3(c) is a stress component excluding $\sigma_{y o}$ which should be increased for yielding of a polycrystal by the pile-up dislocations.

Generally, changes in elastic modulus with temperature and plastic deformation are within about $4 \%$ in the temperature range of room temperature to liquid nitrogen temperature $^{23)}$ and $6-11 \%,{ }^{17,18)}$ respectively. Therefore, the Hall-Petch coefficient, which is linearly proportional to the elastic modulus, ${ }^{24-26)}$ is little changed with temperature $^{27-29)}$ and plastic deformation. ${ }^{30)}$ Due to the thermal component, ${ }^{3,31,32)}$ the critical resolved shear stress of body-centered cubic (BCC) metals increases abruptly with decreasing temperature and increasing strain rate. However, the influence of the thermal component on the critical resolved shear stress of face-centered cubic (FCC) metals is negligible. ${ }^{33)}$ The Hall-Petch relation of Fig. 3(c) is as a result simply shifted upward the high yield strength range with decreasing temperature and with increasing plastic strain and strain rate. The upward shift of the Hall-Petch relation with plastic strain in a $\mathrm{Cu}-\mathrm{Zn}$ alloy ${ }^{30)}$ is consistent with the present expectation. While the original Hall-Petch

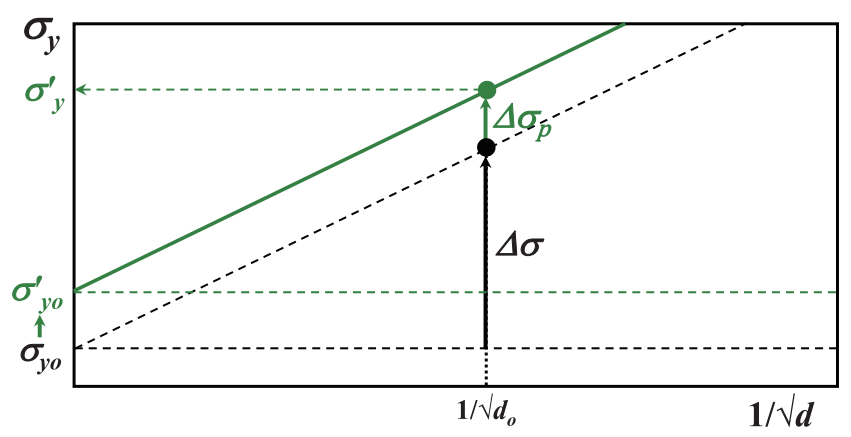

Fig. 4. Schematic Hall-Petch relations before and after plastic deformation. (Online version in color.) 
relation, which has been experimentally obtained, shows a linear relation between the lower yield point $\left(\sigma_{\text {l.y.p. }}\right)$ after yielding and $d^{-1 / 2}$, the above Hall-Petch relation describes the linear relation between the yield strength $\sigma_{y}$ and $d^{-1 / 2}$. Due to some increase in strength by the plastic deformation after yielding, the original Hall-Petch relation is located at some higher position than the present Hall-Petch relation.

\section{Application of Hall-Petch Relation Concept to Deri- vation of Universal Equation Governing Engineer- ing Stress-strain Curves of Polycrystals}

During plastic deformation, the mean shear stress in Fig. 4, which is produced on the slip planes of grains participating in the plastic deformation, can be expressed by

$$
\bar{\tau}=\bar{M} \sigma_{y}^{\prime}=\bar{M}\left(\sigma_{y o}+\Delta \sigma+\Delta \sigma_{p}\right)
$$

where $\bar{M}$ is the mean Schmid factor of the grains participating in the plastic deformation and $\Delta \sigma_{p}$ is an incremental stress component arising from the plastic deformation. As mentioned above, $\sigma_{y}^{\prime}$ of Fig. 4 , is the yield strength of the specimen pre-strained to $e_{p}$. Due to the plastic deformation, $\sigma_{y o}$ increases to $\sigma_{y o}^{\prime}$. The relationship between $\sigma_{y o}$ and $\dot{e}_{c}$ can be obtained from the previous research, ${ }^{3)}$ which is expressed by

$$
\sigma_{y o}=\alpha\left(\dot{e}_{c}\right)^{m}
$$

where $\alpha$ is a coefficient and $m$ is a strain rate exponent. The stress $\sigma_{y o}$ is a function of temperature $e^{34,35)}$ as well as strain rate. $^{3)}$

When the shear stress $\bar{\tau}_{o}$, which produces the average unit velocity of dislocations within the grains participating in the plastic deformation, is approximated to be proportional to the incremental stress component $\Delta \sigma_{p}$, it can be additionally expressed by

$$
\bar{\tau}_{o}=\tau_{o}+\bar{M} \cdot \Delta \sigma_{p}
$$

where $\tau_{o}$ is the shear stress which produces a unit velocity of dislocations in single crystal of the annealed state. ${ }^{19)}$ From Eqs. (8), (9) and (10), the constant strain rate of Eq. (5) is finally expressed by

$$
\dot{e}_{c}=\frac{\dot{e}_{c}}{E} \cdot \frac{d \sigma\left(e_{t}\right)}{d e_{t}}+\frac{1}{2} \bar{\rho}_{m} \boldsymbol{b} \cdot\left(\frac{\sigma_{y o}+\Delta \sigma+\Delta \sigma_{p}}{\tau_{o} / \bar{M}+\Delta \sigma_{p}}\right)^{n}
$$

where $\bar{\rho}_{m}$ is the mean density of mobile dislocations in grains of the polycrystal which participate into the plastic deformation. Equation (11) is very useful for understanding the phenomena observed in the engineering stress-strain curve of Fig. 1.

\section{Changes in Yield Drop Magnitude with Strain Rate, Temperature and Grain Size}

During yield drop after the upper yield strength, the elastic strain rate in Eq. (11) is negative due to $\frac{d \sigma\left(e_{t}\right)}{d e_{t}}$ of the negative sign. In this case, the plastic strain rate is much higher than the constant strain rate. The increase of plastic strain rate higher than constant strain rate and the resultant abrupt decrease of elastic strain rate cause the increase of yield drop magnitude.

The yield drop occurs when slip bands composed of mobile dislocations pass through the tensile specimen body and so the plastic strain rate by the mobile dislocations exceeds the given constant strain rate. In Fig. 5 showing changes in $\tau_{o}$ and $\sigma_{y o}$ with temperature, these stresses increase with decreasing temperature. $\sigma_{y o}$ in Fig. 5(a) is redrawn from the previous researches ${ }^{34-37)}$ and $\tau_{o}$ from the research. ${ }^{19)}$ As shown in Fig. 5(a), the tendency in stress increase with decreasing temperature is much higher in $\sigma_{y o}$. In Fig. 5(b) obtained from Fig. 5(a), the ratio $\sigma_{y o} / \tau_{o}$ in Eq. (11) increases abruptly with decreasing temperature. It is expected that the dependence of $\sigma_{y o} / \tau_{o}$ on strain rate is probably similar to that of $\sigma_{y o} / \tau_{o}$ on temperature, although the related data is not unfortunately found in a literature. Finally, the increase in $\sigma_{y o}$ and $\sigma_{y o} / \tau_{o}$ with decreasing temperature ${ }^{38,39)}$ and with increasing strain rate ${ }^{3)}$ causes the increase of yield drop magnitude. The appearance ${ }^{38)}$ in yield drop of single crystal iron with decreasing Schmid factor and thus increasing $\sigma_{y o}$ may be understood on the concepts described in Fig. 5.

The expectation of Eq. (11) where the yield drop magnitude increases with decreasing grain size is consistent with Fig. 2(a). However, such an expectation is not consistnet in the smaller grain size range of Fig. 2(b). That is, the decrease in plastic strain rate with decreasing grain size after the maximum flow stress in the smaller grain size and the absence of sharp yield drop are probably attributed to the decrease in mobile dislocation density which arises from the higher plastic deformation until the maximum flow stress of Fig. 2(b).
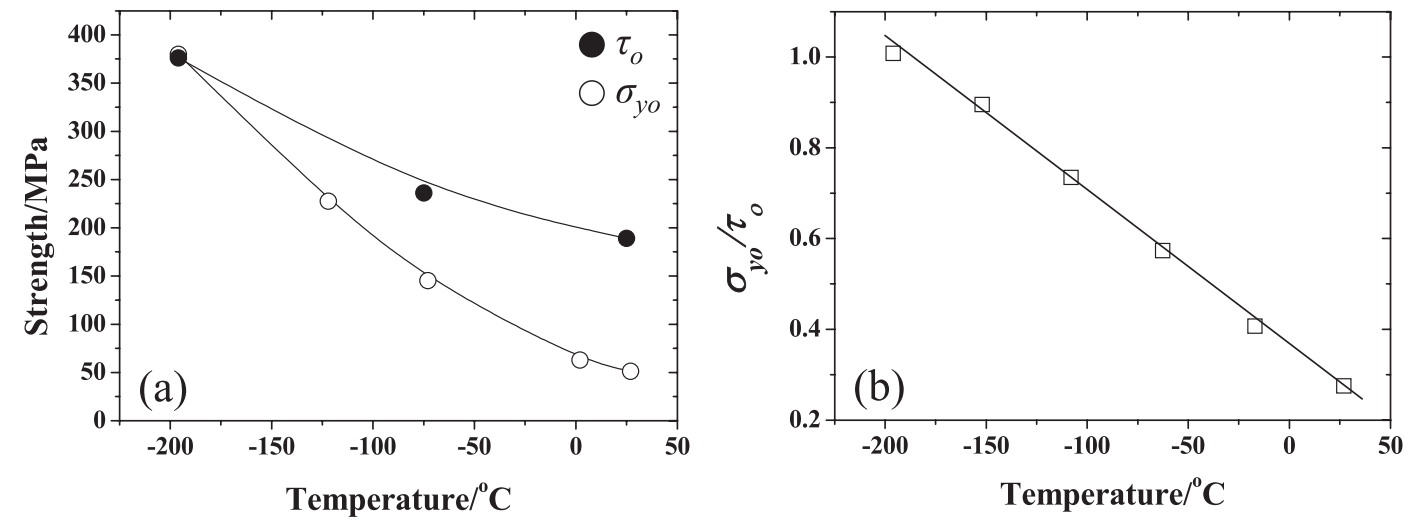

Fig. 5. Changes in $\tau_{o}$ and $\sigma_{y o}$ with temperature (a) and dependence of $\sigma_{y o} / \tau_{o}$ on temperature (b). 


\section{Grain Size and Lüders Strain}

The Lüders strain of Fig. 2(a) increases to a critical grain size $(0.57 \mu \mathrm{m})$ with decreasing grain size. Assuming that the length increase $(\Delta l)$ of Fig. 6 produced by the formation of one Lüders band is same, the total length increase $\left(\Delta l_{t}\right)$ is much larger in the thinner Lüders band even in the same total deformation region $(H)$ of the gage length. Because the Lüders band width $(w)$ is equivalent to the grain size, ${ }^{40-42)}$ the increase in Lüders strain with decreasing grain size to the critical grain size can be attributed to the thinner Lüders band width $(w)$.

It is shown also in Fig. 2(a) that the Lüders strain decreases rather with decreasing grain size after the critical grain size. Even in the same plastic deformation, the

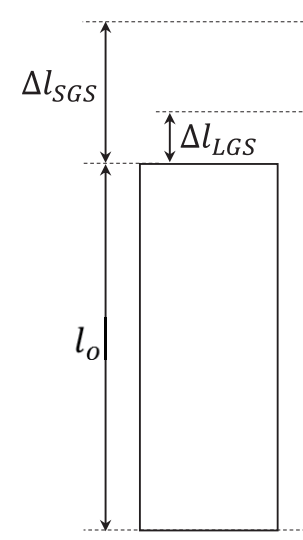

(a)

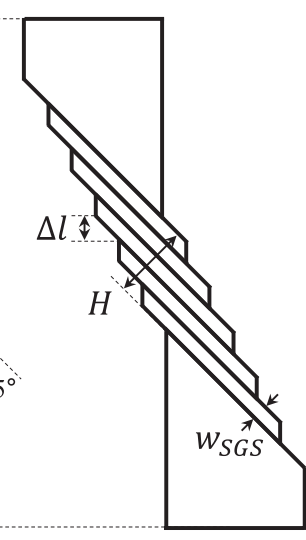

(c)
Fig. 6. Schematic diagrams for explaining the difference of Lüders strain on grain size: (a) before tensile test, (b) the increase of length $\left(\Delta l_{L G S}\right)$ at the larger grain size, and (c) the increase of length $\left(\Delta l_{S G S}\right)$ at the smaller grain size. $l_{o}$ is the initial gage length of the plate specimen. $H$ is the region where the Lüders bands are formed in the gauge length.
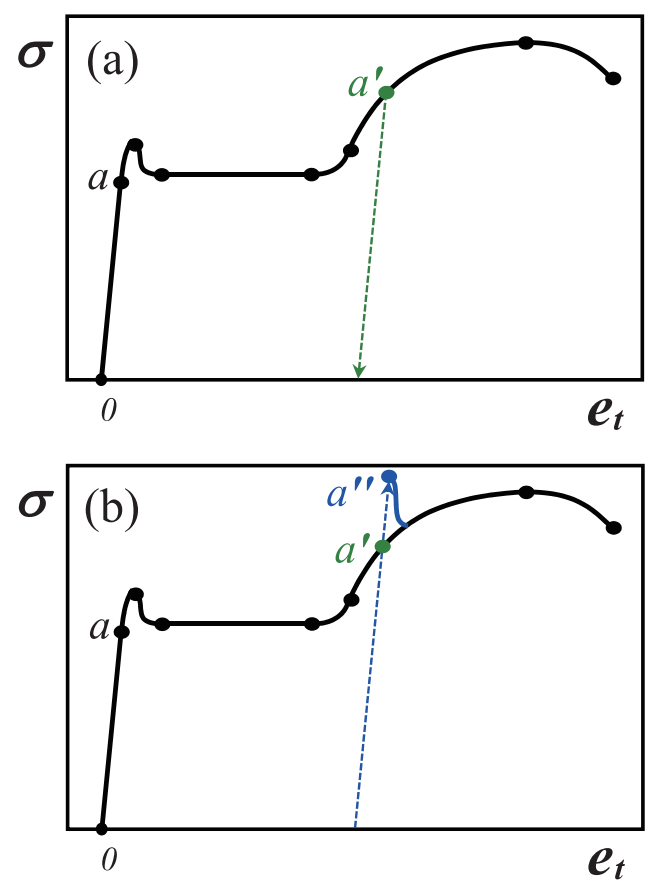

dislocation density increases with decreasing grain size. ${ }^{24,25)}$ With further plastic deformation, the dislocation density is saturated at around $2.5 \times 10^{10} / \mathrm{cm}^{2}$, regardless of the grain size. ${ }^{43,44)}$ The maximum dislocation density appears at the necking point during tensile test, after which the dislocation density decreases with increasing plastic strain. ${ }^{45}$ The necking phenomenon has been well understood in the light of thermal softening and dynamic recrystallization which occur during tensile test. ${ }^{46-49)}$ In Fig. 2(a), the decrease in Lüders strain with decreasing grain size is therefore attributed to the faster saturation in dislocation density with decreasing grain size after the critical grain size. From Figs. 2(a) and 2(b), the low ductility of nanocrystalline materials ${ }^{8-16)}$ is therefore their intrinsic property.

\section{Work Hardening and Strain Aging in Polycrystals}

From Eq. (11), the plastic strain rate increases with decreasing grain size and thus with increasing $\Delta \sigma$. This results in the decrease in elastic strain rate and the consequent decrease in overall work hardening rate, as shown in Fig. 2(b). Materials of a high dislocation density show generally no yield drop. ${ }^{50,51)}$ No yield drop is probably attributed to the much lower mobile dislocation density in the plastic strain rate of Eq. (11).

Figure 7 shows schematic diagrams for explaining the yield drop in strain-aged polycrystals. In Fig. 7(a), the load is released from the tensile test specimen after a tensile specimen is plastically deformed to a stress level $a^{\prime}$. As mentioned above, the stress level $a^{\prime}$ is the yield strength of the pre-strained specimen, as shown in the Hall-Petch relation of Fig. 7(c). After pre-straining, $\sigma_{y o}$ in the Hall-Petch relation is increased to $\sigma_{y o}^{\prime}$. When strain aging is given to the pre-strained specimen, $\sigma_{y o}^{\prime}$ increases to $\sigma^{\prime \prime}{ }_{y o}$ due to the Cottrell drag stress $\sigma_{C}$, as shown in the Hall-Petch relation of Figs. 7(b) and 7(c). The yield strength of the strain-aged

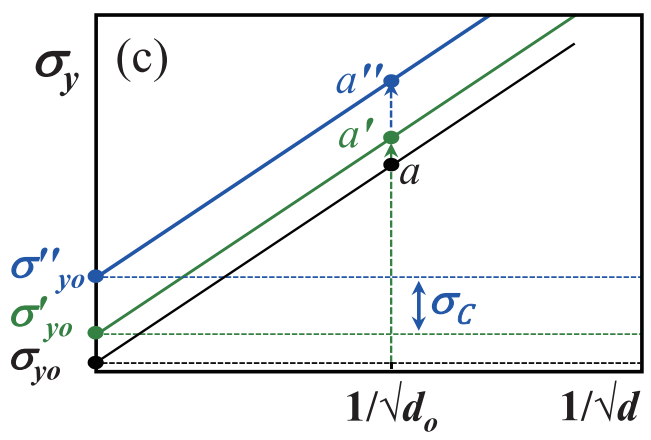

Fig. 7. Schematic diagrams for understanding the strain aging: (a) pre-straining, (b) tensile test after strain aging, and (c) Hall-Petch relations corresponding to the initial state of tensile specimen, the pre-strained specimen, and the strain-aged specimen. (Online version in color.) 
specimen increases from $a^{\prime}$ to $a^{\prime \prime}$. During tensile test on the strain-aged specimen, mobile dislocations move to pile up at grain boundaries, when the applied stress exceeds $\sigma^{\prime \prime}{ }_{y o}$ and the mobile dislocations are concurrently released from the Cottrell atmosphere. The strain-aged specimen is finally yielded at the yield strength $a^{\prime \prime}$. The flow stress decreases with further plastic deformation and follows the engineering stress-strain curve, as shown in Fig. 7(b). As a result, the yield drop in the strain-aged polycrystals does not occur when mobile dislocations are released from the Cottrell atmosphere but when the applied stress exceeds the yield strength of the strain-aged specimen after escaping the Cottrell atmosphere. However, The yield drop in the strain-aged single crystals occurs when mobile dislocations are released from the Cottrell atmosphere.

\section{Conclusions}

A universal equation governing engineering stress-strain curves of polycrystals has been derived. Various phenomena such as yielding, yield drop, Lüders strain, work hardening, and strain aging are clearly understood using the equation including the Hall-Petch relation concept. The results are as follows.

(1) Yielding occurs when the shear stress created at the front of pile-up dislocations in specific grains showing the Schmid factor of 0.5 exceeds the theoretical shear stress between the slip planes of neighboring grains.

(2) The yield drop magnitude increases with decreasing temperature and grain size and with increasing strain rate. The increase in yield drop magnitude with decreasing temperature and increasing strain rate is due to the increase in ratio of the minimum applied stress necessary for the movement of mobile dislocations to shear stress producing the unit velocity of mobile dislocations. This is because the increase of the ratio finally causes the increase of plastic strain rate. The increase in yield drop magnitude with decreasing grain size is attributed to the increase of pile-up stress which causes the plastic strain rate.

(3) The increase in Lüders strain with decreasing grain size can be attributed to the decrease in Lüders band width with decreasing grain size.

(4) Even at the same plastic strain, the dislocation density is higher at the smaller grain size. The saturation level of dislocation density, which is observed at the necking point during deformation, is similar, regardless of the grain size. the decrease in ductility with decreasing grain size is due to the faster saturation of dislocation density during deformation. Therefore, the low ductility of nanocrystalline materials is their intrinsic property.

(5) The work hardening behavior becomes prominent with increasing grain size, due to the decrease in plastic strain rate.

(6) Strain-aged single crystals or polycrystals show a yield drop phenomenon. The yield drop in the strain-aged single crystals occurs when mobile dislocations are released from the Cottrell atmosphere. However, the yield drop in the strain-aged polycrystals does not occur when mobile dislocations are released from the Cottrell atmosphere but when the applied stress exceeds the yield strength of the strain-aged specimen after escaping the Cottrell atmosphere.

\section{REFERENCES}

1) R. L. Miller: Metall. Trans., 3 (1972), 905

2) N. Tsuji, Y. Ito, Y. Saito and Y. Minamino: Scr. Mater., 47 (2002), 893.

3) J. Harding: Acta Metall., 17 (1969), 949.

4) A. H. Cottrell: Dislocations and Plastic Flow in Crystals, Oxford University Press, London, (1953).

5) W. G. Johnston: J. Appl. Phys., 33 (1962), 2716.

6) W. G. Johnston and J. J. Gilman: J. Appl. Phys., 30 (1959), 129

7) W. Lüders: Dinglers Polytech. J., 155 (1860), 18.

8) W. P. Anthony: Acta Metall., 25 (1977), 83.

9) G. W. Nieman, J. R. Weertman and R. W. Sigel: Nanostructured Mater., 1 (1992), 185

10) J. R. Weertman: Mater. Sci. Eng. A, 166 (1993), 161.

11) P. C. Sanders, C. J. Youngdahl and J. R. Weertman: Mate

12) P. G. Sanders, J. A. Eastman and J. R. Weertman: Acta Mater., 45 (1997), 4019.

13) Y. Wang, M. Chen, F. Zhou and E. Ma: Nature, 419 (2002), 912.

14) X. Huang, N. Hansen and N. Tsuji: Science, 312 (2006), 249.

15) M. A. Meyers, A. Mishra and D. J. Benson: Prog. Mater. Sci., 51 (2006), 427.

16) H. Li, J. Zhou, R. Zhu and X. Ling: Mater. Design, 31 (2010), 1003.

17) J. Benito, J. M. Manero, J. Jorba and A. Roca: Metall. Mater. Trans. A, 36 (2005), 3317.

18) H. M. Ledbetter and S. A. Kim: Mater. Sci. Eng. A, 101 (1988), 87.

19) J. J. Gilman and W. G. Johnson: J. Appl. Phys., 31 (1960), 687.

20) G. T. Hahn: Acta Metall., 10 (1962), 727.

21) E. Smith and J. T. Barnby: Met. Sci., 1 (1967), 56.

22) N. T. Barrett: The Principles of Engineering Materials, Prentice-Hall Inc., NJ., (1973).

23) J. A. Rayne and B. S. Chandrasekhar: Phys. Rev., 122 (1961), 1714

24) H. Conrad, S. Feuerstein and L. Rice: Mater. Sci. Eng., 2 (1967), 157.

25) D. T. Narutani and J. Takamura: Acta Metall., 39 (1991), 2037.

26) M. Etou, S. Fukushima, T. Sasaki, Y. Haraguchi, K. Miyata, M. Wakita, T. Tomida, N. Imai, M. Yoshida and Y. Okada: ISIJ Int., 48 (2008), 1142.

27) N. J. Petch: Philos. Mag., 1 (1956), 186.

28) J. Heslop and N. J. Petch: Philos. Mag., 1 (1956), 866.

29) N. J. Petch: Philos. Mag., 3 (1958), 1089.

30) R. Armstrong, I. Codd, R. M. Douthwaite and N. J. Petch: Philos. Mag., 7 (1962), 45.

31) G. R. Speich: Physical Metallurgy of Steels, The Metallurgy Society of AIME, New York, (1981).

32) D. F. Stein, J. R. Low and A. U. Seybolt: Acta Metall., 11 (1963), 1253.

$33)$ R. Liang and A. S. Khan: Int. J. Plast., 15 (1999), 963.

34) B. Jaoul and D. Gonzalez: J. Mech. Phys. Sol., 9 (1961), 16.

$35)$ R. P. Steijn and R. M. Brick: Trans. Am. Soc. Met., 46 (1954), 1406.

36) J. J. Cox, G. T. Horne and R. F. Mehl: Trans. Am. Soc. Met., 49 (1957), 118.

37) N. P. Allen, B. E. Hopkins and J. E. McLennan: Proc. Roy. Soc. A, 234 (1956), 221.

38) D. J. Dingley and D. McLean: Acta Metall., 15 (1967), 885.

39) W. A. Spitzig and A. Keh: Acta Metall., 18 (1970), 611.

40) E. O. Hall: Proc. Phys. Soc. B, 64 (1951), 742

$41)$ F. T. Humble, J. G. Speer and D. K. Matlock: SAE Int., 1 (2004), 8.

42) I. Gaal, A. L. Toth, L. Uray and P. Harmat: Int. J. Ref. Met. Hard Mater., 24 (2006), 325.

43) H. Conrad and B. Christ: Report No. TDR-169(3240-11)TN-6, Aerospace Cor., California, (1963), 1.

44) W. C. Leslie: The Physical Metallurgy of Steels, McGraw-Hill international company, New York, (1982), 6.

45) P. Schall, M. Feuerbacher, M. Bartsch, U. Messerschmidt and K. Urban: Philos. Mag. Lett., 79 (1999), 785.

46) D. Rittel and Z. Wang: Mech. Mater., 40 (2008), 629.

47) S. Osovski, Y. Nahmany, D. Rittel, P. Landaub and A. Venkert: Scr. Mater, 67 (2012), 693.

48) S. Osovski, D. Rittel and A. Venkert: Mech. Mater., 56 (2013), 11

49) J. A. Rodriguez-Martinez, G. Vadillo, D. Rittel, R. Zaera and J. Fernández-Sáez: Mech. Mater., 80 (2015), 41.

50) M. Kumagai, M. Imafuku and S. I. Ohya: ISIJ Int., 54 (2014), 206.

51) E. Rafiee, M. Farzam, M. A. Golozar and A. Ashraft: ISRN Corrosion., 2013 (2013), 1. 